\title{
Sensitivity Analysis Using Simple Additive Weighting Method
}

\author{
Wayne S. Goodridge \\ Department of Computing and Information Technology, The University of the West Indies, Trinidad and Tobago, W.I \\ E-mail: wayne.goodridge@sta.uwi.edu
}

\begin{abstract}
The output of a multiple criteria decision method often has to be analyzed using some sensitivity analysis technique. The SAW MCDM method is commonly used in management sciences and there is a critical need for a robust approach to sensitivity analys is in the context that uncertain data is often present in decision models. Most of the sensitivity analysis techniques for the SAW method involve Monte Carlo simulation methods on the initial data. These methods are computationally intensive and often require complex software. In this paper, the SAW method is extended to include an objective function which makes it easy to analyze the influence of specific changes in certain criteria values thus making easy to perform sensitivity analysis.
\end{abstract}

Index Terms-SAW, Sensivity Analysis, MCDM, Objective Function.

\section{INTRODUCTION}

Multiple criteria decision making (MCDM) is used when a decision maker wishes to find the best alternative or rank a list of alternatives in a rational and efficient manner when multiple decision criteria are involved. One of the earliest MCDM methods used is the simple additive weighting (SAW) [1],[2],[3],[4]. The SAW method is largely used in the management discipline for selection of suppliers, projects and facility locations [5], [6], [7]. However, the SAW method is less used in the research community in favour of other methods such as the Analytic Hierarchy Process (AHP) [8], [9], [10] and Technique for Order of Preference by Similarity to Ideal Solution (TOPSIS) [7],[8],[11],[12].

In a MCDM problem there are four components, namely: (1) alternatives, (2) criteria, (3) relative importance (weights) of each criterion, and (4) criterion values for each alternative. A decision table consisting of these four components is shown in Table 1. The decision table shows alternatives, $A_{i}(1 \leq i \leq n)$, criteria, $C_{j}$ $(1 \leq j \leq m)$, weights of criteria, $w_{j}(1 \leq j \leq m)$ and the measures of performance of alternatives, $x_{i j}$.

There are four main steps in the formulation of MCDM problems:

1. Determining the relevant criteria and alternatives.

2. Ascertain the measures of performance of the alternatives in terms of the selected criteria.

3. Attaching numerical measures to the relative importance (criteria weights)

4. Application of an algorithm on the numerical values to determine a ranking of each alternative.

Quantitative decision methods like the SAW and TOPSIS takes the decision table given in Table 1 as input and produces a ranked set of alternatives. Although this output could be useful to decision makers many times decision makers wish to analyze the impact of changes to criteria data given the fact that some of the data in the first place may be uncertain. This process is called sensitivity analysis (SA) [13],[15] and helps the decision maker compare different scenarios and their potential outcomes based on changing conditions. In management decisions involving selection of suppliers, projects and procurement of assets often involve sensitivity analysis. Since SAW is a commonly used MCDM method in the management discipline it is important to have a robust approach to sensitivity analysis as it relates to the SAW method.

Table 1. Components of a Decision Problem

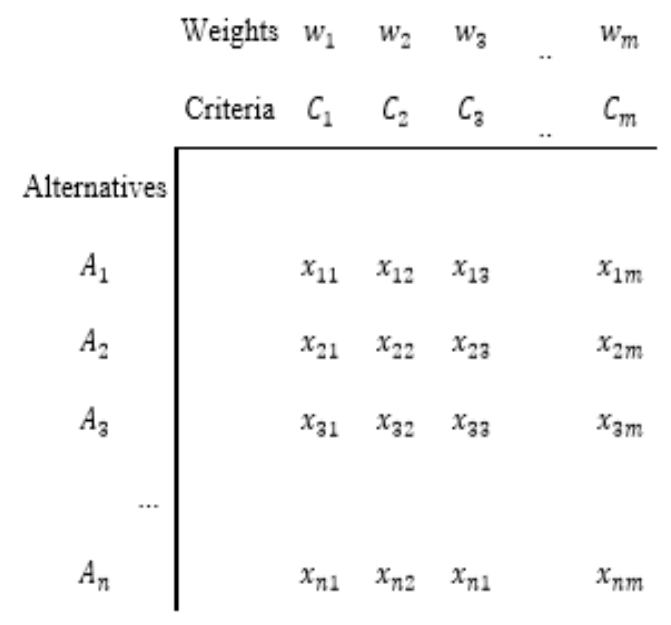

Linear Programming (LP) provides a natural framework for SA which is typically done after the optimal solution to the problem is determined. The SA is said to be a post-optimality step. The decision maker wants to see how sensitive an optimal alternative is to changes in the constraints and the objective function. However, research on SA in MCDM methods is limited 
with respect to post-optimality step approaches.

To capture the advantages that the LP method has as it relates to $\mathrm{SA}$, we proposed an extension of the SAW method called Sensitive - Simple Additive Weights (SSAW) method which due to its use of an objective function provides a framework for a novel approach to sensitivity analysis which does not involve weight changes or changes to alternatives criteria values. Like the LP approach, selected changes are made to the objective function and the impact of the optimal alternative is studied.

This paper is organized as follows. Section II gives an overview of existing approaches to sensitivity analys is in MCDM methods. The SAW method and the S-SAW method which can be seen as an extension to the SAW method are described in Sections III and IV respectively. The S-SAW method for sensitivity analysis is discussed in Section $\mathrm{V}$ and examples to show how the new approach to sensitivity analysis works are provided. Section VI introduces mathematical insights of how the S-SAW method works. Finally, the last section presents the conclusion of the proposed method.

\section{EXISTING APPROACHES TO SENSITIVITY ANALYSIS}

A lot of research on SA as it relates to MCDM methods is focused on the assessment and influence of criteria weights [14], [15], [16] with the goal of determining how critical each criterion is. The conclusions of [14] suggest that the most sensitive decision criterion is the one with the highest weight, if weight changes are measured in relative terms. This approach is limited because it does not address the combine impact of selected criteria on the ranked alternatives.

The authors of [14] presented a complex sensitivity analysis approach which deals with the change of the values of the alternatives against the criteria. A fixed set of criteria weights are used in the sensitivity analysis process to determine the range of values that a given criterion can have without altering the rank order of the alternatives given that all other criteria weights are kept constant. This approach is useful but is very involved and requires a lot of effort to determine the change to a given criterion for a particular alternative which would invoke a rank change in that alternative.

SA in MCDM is often not done as a post-optimality step like in the LP approach. Many approaches [14], [18] use Monte Carlo Simulations methods for the generation of data sets based on the initial data. These data sets are then used to study how slight variations of the initial criteria data values result in the changes of ranking of the optimal alternative. In this way, decision makers will know what impact each criterion has on the optimal alternative. The main drawback of this approach is that it is computational intensive.

When Monte Carlo methods are used with MCDM techniques [3],[18],[19] the result is that a definitive answer is not provided due to the fact that the inputs are stochastic in nature. As a result these approaches can lead to complex interpretations about the risks associated with solutions.

Considering the reviewed gaps in sensitivity analysis involving Monte Carlo MCDM techniques and the drawbacks of the post-optimality approaches of [14], [15], [16] this paper introduces a post-optimality approach which uses an objective function where selected changes are made to the objective function and the impact of the optimal alternative is studied.

\section{SAW}

The SAW MCDM method is very simple and popular. The method takes the decision table shown in Table 1 as input and produces a ranked set of alternatives. There are two types of evaluation criteria: benefit and cost criteria. A benefit criterion means that the higher the value of the criterion the better the value is for a given alternative. For example, if a customer has a choice between two Internet Service Providers (ISPs) A [bandwidth $=500 \mathrm{Mbps}$, price $=\$ 200$ ] and $\mathrm{B}$ [bandwidth $=200 \mathrm{Mbps}$, price $=\$ 50$ ], and the criteria are price and bandwidth, then bandwidth is a benefit criterion since a customer would want higher bandwidths. On the other hand, price is a cost criterion since a customer would want to minimize the price he pays for the service.

A key part of the SAW method is the normalization process, which takes criteria performance values and transform them to dimensionless units. The larger the normalized value becomes, the more preference it has. There are basically two approaches to normalization:

1. Distance-Based Normalization Methods [20] - this invloves measuring the eucludian distance from origin for each criterion

2. Proportion Based Normalization Methods - the proportion of difference between performance value of the alternative and the worst performance value to difference the between the best and the worst performance values [21]

The SAW method can be used with different normalization procedures. In this paper, we use the Linear Scale Transformation, MaxMin method [2], [22] which is a proportion based normalization method. Using this method the decision matrix given in Table 1 is normalized by equations 1 and 2 :

$$
\begin{aligned}
& z_{i j}=\frac{\left(x_{i j}-b_{j j}\right)}{\left(a_{j}-b_{j}\right)}, i=1_{w \ldots,} n ; j \in \Omega_{b} \\
& z_{i j}=\frac{\left(a_{j}-x_{i j}\right)}{\left(a_{j}-b_{j j}\right)}, i=1_{m \ldots,} n_{;} j \in \Omega_{c}
\end{aligned}
$$

where $z_{i j}$ are normalized criterion values, $a_{j}$ is the $\max \left(x_{i j}\right)$ for criterion $j$ and, $b_{j}$ is the $\min \left(x_{i j}\right)$ for criterion $\bar{l}, \Omega_{b}$ and $\Omega_{c}$ are, respectively, the sets of benefit and cost criteria. Associated with each criterion column $j$ in the decision matrix $z_{i j}$ is weighting $w_{j}$ such that 
$\sum_{j=1}^{m} w_{j}=1$. The overall assessment of each alternative is computed by Equation 3. The greater the value $y_{i}$ means that the alternative is ranked higher.

$$
y_{i}=\sum_{j=1}^{m} z_{i j} w_{j}, i=1_{x \ldots s} n
$$

\section{S-SAW METHOD}

The S-SAW method can be seen as an extension of the SAW method. The major difference is that the S-SAW allows the decision maker to define an objective function which governs the optimization goals of each criterion. This concept of optimization will be formally defined in Section V1. The first step in the S-SAW method is to normalized the decision matrix given in Table 1 by using equations 4 and 5 :

$$
\begin{aligned}
& z_{i j}=\frac{2\left(x_{i j}-b_{j}\right)}{\left(a_{j j}-b_{j}\right)}-1, i=1, \ldots, n ; j \in \Omega_{b} \\
& z_{i j}=1-\frac{2\left(x_{i j}-b_{j}\right)}{\left(a_{j}-b_{j j}\right)}, i=1_{, \ldots,} n_{i j} \in \Omega_{c}
\end{aligned}
$$

where $z_{i j}$ are normalized criterion values, $a_{j}$ is the $\max \left(x_{i j}\right)$ for criterion $j$ and, $b_{j}$ is the $\min \left(x_{i j}\right)$ for criterion $j, \Omega_{b}$ and $\Omega_{c}$ are, respectively, the sets of benefit and cost criteria. The $z_{\mathrm{i} j}$ matrix has dimensionless values in the range $[-1,1]$. The function which transforms the matrix $x_{i j}$ to $z_{i j}$ can be any monotonic continuous increasing or decreasing function whose range exists on $[-1,1]$.

The proposed S-SAW method has a $F^{*}$ function which accepts a criterion $j$ and maps it to the set $\{-1,0,1\}$. That is $F^{*}(j) \in\{-1,0,1\}$ where $j \in\{1,2, \ldots, m\}$. The value for $j$ is decided by the decision maker and $F^{*}(j)$ is called the objective coefficient.

Key to the S-SAW method is Equation 6. After the matrix multiplication process the $y_{i}$ position with the largest value represents the preferred alternative. An analysis of the S-SAW method will be given in Section VI.

$$
\left[\begin{array}{llll}
z_{11} & z_{12} & \ldots & z_{1 m} \\
z_{21} & z_{22} & \ldots & z_{2 m} \\
\ldots & \ldots & \ldots & \ldots \\
z_{n 1} & z_{n 2} & \ldots & z_{n m}
\end{array}\right]\left[\begin{array}{l}
w_{1} F^{*}(1) \\
w_{2} F^{*}(2) \\
\ldots \\
w_{m} F^{*}(m)
\end{array}\right]=\left[\begin{array}{l}
y_{1} \\
y_{2} \\
\ldots \\
y_{n}
\end{array}\right]
$$

The S-SAW algorith m has a special property which the SAW algorithm does not possess. This is the ability for the decision maker to express which criteria are to be optimized, ignored or minimized. This special property is achieved via the use of an objective function which should not be confused with criteria weights. In other words, the decision maker has more power to customize the type of alternatives the decision system produces. The SAW only allows the decision maker to consider criteria weightings.

\section{A. S-SAW Examples}

Table 2 shows a decision problem with 5 alternatives and 5 decision criteria. The decision matrix before normalization shows the $a_{j}$ and $b_{j}$ values for each criterion. Using Equations 4 and 5 the data is normalized and then Equation 6 is used to produce $y_{\mathrm{i}}$. The rank order of the alternatives is shown in the column labelled "Rank". Table 2 has a value of 1 for the optimization coefficient value of each criterion, meaning that alternatives which "optimize" values for all or most criteria will be ranked higher.

The data in Table 3 is similar to Table 2 with the only difference that each optimization coefficient value is set to -1 , meaning that alternatives wth minimum values for all or most criteria will be ranked higher. Notice that the rank order in Table 2 is $A_{1} \geq A_{2} \geq A_{4} \geq A_{2} \geq A_{5}$ and

\begin{tabular}{|c|c|c|c|c|c|c|c|c|c|c|c|c|}
\hline \multirow[b]{2}{*}{ Criteria } & \multicolumn{5}{|c|}{ Decision Matrix } & \multirow[b]{2}{*}{$C_{1}$} & \multicolumn{5}{|c|}{ Normalized Decision Matrix } & \\
\hline & $C_{1}$ & $C_{2}$ & $C_{\mathrm{a}}$ & $C_{4}$ & $C_{5}$ & & $C_{2}$ & $C_{\mathrm{a}}$ & $C_{4}$ & $C_{5}$ & $y_{i}$ & \\
\hline$w_{j}$ & 0.41 & 0.013 & 0.30 & 0.06 & 0.22 & 0.41 & 0.013 & 0.30 & 0.06 & 0.22 & & \\
\hline Opt. Goal & & & & & & 1.00 & 1.00 & 1.00 & 1.00 & 1.00 & & Rank \\
\hline$A_{1}$ & 0.36 & 0.25 & 0.29 & 0.30 & 0.32 & 0.98 & 0.63 & 0.95 & 0.83 & 0.73 & 0.91 & 1 \\
\hline$A_{2}$ & 0.36 & 0.28 & 0.04 & 0.09 & 0.02 & 1.00 & 1.00 & -1.00 & -0.82 & -1.00 & -0.13 & 3 \\
\hline$A_{a}$ & 0.03 & 0.17 & 0.30 & 0.22 & 0.26 & -1.00 & -0.14 & 0.95 & 0.22 & 0.41 & -0.03 & 4 \\
\hline$A_{4}$ & 0.16 & 0.20 & 0.30 & 0.07 & 0.03 & -0.19 & 0.13 & 1.00 & -1.00 & -0.92 & -0.04 & 2 \\
\hline$A_{5}$ & 0.10 & 0.09 & 0.08 & 0.32 & 0.37 & -0.58 & -1.00 & -0.66 & 1.00 & 1.00 & -0.17 & 5 \\
\hline$a_{j}$ & 0.36 & 0.28 & 0.30 & 0.32 & 0.37 & & & & & & & \\
\hline$b_{j}$ & 0.03 & 0.09 & 0.04 & 0.07 & 0.02 & & & & & & & \\
\hline
\end{tabular}
the rank order in Table 3 is $A_{5} \geq A_{2} \geq A_{4} \geq A_{9} \geq A_{1}$ which is a complete reversal of the order.

Table 2. Example of S-SAW operation under optimization function $F^{\wedge *}(j)=1$ for $j=1, \ldots, m$ 
Table 3. Example of S-SAW operation under optimization function $F^{\wedge *}(j)=-1$ for $j=1, \ldots, m$

\begin{tabular}{|c|c|c|c|c|c|c|c|c|c|c|c|c|}
\hline & Decis & Matrix & & & & & Norm & dDeci & Matrix & & & \\
\hline Criteria & $C_{1}$ & $C_{2}$ & $C_{a}$ & $C_{4}$ & $C_{5}$ & $C_{1}$ & $C_{2}$ & $C_{\mathrm{a}}$ & $C_{4}$ & $C_{5}$ & $y_{i}$ & \\
\hline$w_{i}$ & 0.41 & 0.013 & 0.30 & 0.06 & 0.22 & 0.41 & 0.013 & 0.30 & 0.06 & 0.22 & & \\
\hline Opt. Goal & & & & & & -1.00 & -1.00 & -1.00 & -1.00 & -1.00 & & Rank \\
\hline$A_{1}$ & 0.36 & 0.25 & 0.29 & 0.30 & 0.32 & \begin{tabular}{|l|}
0.98 \\
\end{tabular} & 0.63 & \begin{tabular}{|l|}
0.95 \\
\end{tabular} & \begin{tabular}{|l|}
0.83 \\
\end{tabular} & \begin{tabular}{|l|}
0.73 \\
\end{tabular} & \begin{tabular}{|l|}
0.17 \\
\end{tabular} & \begin{tabular}{|l|}
5 \\
\end{tabular} \\
\hline$A_{2}$ & 0.36 & 0.28 & 0.04 & 0.09 & 0.02 & 1.00 & 1.00 & -1.00 & -0.82 & -1.00 & 0.13 & 2 \\
\hline$A_{a}$ & 0.03 & 0.17 & 0.30 & 0.22 & 0.26 & -1.00 & $\mid-0.14$ & 0.95 & 0.22 & 0.41 & 0.04 & 4 \\
\hline$A_{4}$ & 0.16 & 0.20 & 0.30 & 0.07 & 0.03 & \begin{tabular}{|l|}
-0.19 \\
\end{tabular} & 0.13 & 1.00 & -1.00 & -0.92 & 0.03 & 3 \\
\hline$A_{5}$ & 0.10 & 0.09 & 0.08 & 0.32 & 0.37 & -0.58 & $\mid-1.00$ & \begin{tabular}{|l|}
-0.66 \\
\end{tabular} & 1.00 & 1.00 & $\mid-0.91$ & 1 \\
\hline$a_{j}$ & 0.36 & 0.28 & 0.30 & 0.32 & 0.37 & & & & & & & \\
\hline$b_{j}$ & 0.03 & 0.09 & 0.04 & 0.07 & 0.02 & & & & & & & \\
\hline
\end{tabular}

Table 4. Example of SAW operation. There is no concept of an optimization

\begin{tabular}{|l|l|l|l|l|l|l|l|l|l|l|l|l|l|}
\hline & \multicolumn{9}{|l}{ Decision Matrix } & \multicolumn{3}{l|}{ Normalized Decision Matrix } & \multicolumn{1}{l|}{} \\
\hline Criteria & $C_{1}$ & $C_{2}$ & $C_{\mathrm{a}}$ & $C_{4}$ & $C_{5}$ & $C_{1}$ & $C_{2}$ & $C_{\mathrm{a}}$ & $C_{4}$ & $C_{5}$ & $y_{\mathrm{i}}$ & \\
\hline$w_{j}$ & 0.41 & 0.013 & 0.30 & 0.06 & 0.22 & 0.41 & 0.013 & 0.30 & 0.06 & 0.22 & & \\
\hline$A_{1}$ & 0.36 & 0.25 & 0.29 & 0.30 & 0.32 & 0.99 & 0.81 & 0.98 & 0.91 & 0.87 & 0.95 & 1 \\
\hline$A_{2}$ & 0.36 & 0.28 & 0.04 & 0.09 & 0.02 & 1.00 & 1.00 & 0.00 & 0.09 & 0.00 & 0.43 & 3 \\
\hline$A_{a}$ & 0.03 & 0.17 & 0.30 & 0.22 & 0.26 & 0.00 & 0.43 & 0.97 & 0.61 & 0.71 & 0.48 & 4 \\
\hline$A_{4}$ & 0.16 & 0.20 & 0.30 & 0.07 & 0.03 & 0.40 & 0.57 & 1.00 & 0.00 & 0.04 & 0.48 & 2 \\
\hline$A_{5}$ & 0.10 & 0.09 & 0.08 & 0.32 & 0.37 & 0.21 & 0.00 & 0.17 & 1.00 & 1.00 & 0.43 & 5 \\
\hline$a_{j}$ & 0.36 & 0.28 & 0.30 & 0.32 & 0.37 & & & & & & & \\
\hline$b_{j}$ & 0.03 & 0.09 & 0.04 & 0.07 & 0.02 & & & & & & & \\
\hline
\end{tabular}

Table 5. Examples of how the S-SAW method is used to calculate the Sensivitiy measures: MIRR and LIRR

\begin{tabular}{|l|l|l|l|l|l|l|l|l|}
\hline & $C_{1}$ & $C_{2}$ & $C_{\mathrm{a}}$ & $C_{4}$ & $C_{5}$ & Rank Order & Change? & Ratio \\
\hline$w_{j}$ & 0.41 & 0.013 & 0.3 & 0.06 & 0.22 & & & \\
\hline$F^{*}(j)=1$ & 1 & 1 & 1 & 1 & 1 & $A_{1} \geq A_{a} \geq A_{4} \geq A_{2} \geq A_{5}$ & & \\
\hline$F^{*}(p)=-1$ & -1 & 1 & 1 & 1 & 1 & $A_{4} \geq A_{5} \geq A_{1} \geq A_{a} \geq A_{2}$ & Yes & $\begin{array}{l}1 \mid 5 \\
(\mathrm{MIRR})\end{array}$ \\
\hline$F^{*}(j)=1$ & 1 & 1 & 1 & 1 & 1 & $A_{1} \geq A_{a} \geq A_{4} \geq A_{2} \geq A_{5}$ & & \\
\hline$F^{*}(p)=-1$ & 1 & -1 & 1 & 1 & 1 & $A_{1} \geq A_{5} \geq A_{a} \geq A_{2} \geq A_{4}$ & No & \\
\hline$F^{*}(p)=-1$ & 1 & -1 & 1 & -1 & 1 & $A_{1} \geq A_{4} \geq A_{2} \geq A_{2} \geq A_{5}$ & No & \\
\hline$F^{*}(p)=-1$ & 1 & -1 & 1 & -1 & -1 & $A_{1} \geq A_{a} \geq A_{4} \geq A_{2} \geq A_{5}$ & No & \\
\hline$F^{*}(p)=-1$ & 1 & -1 & -1 & -1 & -1 & $A_{2} \geq A_{1} \geq A_{5} \geq A_{a} \geq A_{4}$ & Yes & $\begin{array}{l}4 \mid 5 \\
(\text { LIRR })\end{array}$ \\
\hline
\end{tabular}

The SAW method is a special case of the S-SAW method where the optimization coefficient value of each criterion is set to 1 . Table 4 shows the SAW method output for the same data as in Table 2. The main difference is that the SAW method normalization matrix values exists on $[0,1]$ rather than $[-1,1]$. The final ranking is $A_{1} \geq A_{2} \geq A_{4} \geq A_{2} \geq A_{5}$. However, the SAW method does not have an optimization option for criteria where the decision maker can have an influence on the rank order of the alternatives based on his optimization preferences.

\section{SENSITIVITY ANALYSIS WITH S-SAW}

The approach to sensitivity analysis in this paper focuses on how a collection of criteria impact on the optimal alternative. The objective coefficient for each

criterion in the collection is set to either 1 or -1 until the preferred optimal alternative is no longer optimal. Consider the following definitions:

Most Important Resistant (MIR) Criteria Set Given a preferred alternative $A_{\hat{i}}$ and a set of criteria $C=\{1,2, \ldots, m\}$ where $F^{*}(j)=1$ for all criteria $j \in C$, the MIR criteria set $M \subset C$ is the smallest set of criteria where $F^{*}(p)=-1 p \in M$ that will invoke a change in the rank order such that $A_{i}$ is no longer preferred alternative. The criteria $\mathrm{p}$ are ordered from highest criterion to lowest criterion weight.

Least Important Resistant (LIR) Criteria Set Given a preferred alternative $A_{\hat{i}}$ and a set of criteria $C=\{1,2, \ldots, m\}$ where $F^{*}(j)=1$ for all criteria $j \in C$, the LIR criteria set $M \subset C$ is the smallest set of criteria where $F^{*}(p)=-1, p \in M$ that will invoke a change in the rank order such that $A_{i}$ is no longer preferred alternative. The criteria $\mathrm{p}$ are ordered from lowest criterion to highest criterion weight. 
Table 6. Modified Decision Matrix

\begin{tabular}{|l|l|l|l|l|l|}
\hline & Decision Matrix & $C_{2}$ & $C_{\mathrm{a}}$ & $C_{5}$ \\
\hline Criteria & $C_{1}$ & 0.013 & 0.30 & 0.06 & 0.22 \\
\hline$w_{j}$ & 0.41 & & & & \\
\hline Opt. Goal & & 0.25 & 0.29 & 0.30 & 0.32 \\
\hline$A_{1}$ & 0.29 & 0.28 & 0.04 & 0.09 & 0.02 \\
\hline$A_{2}$ & 0.03 & 0.17 & 0.30 & 0.22 & 0.26 \\
\hline$A_{a}$ & 0.30 & 0.20 & 0.30 & 0.07 & 0.03 \\
\hline$A_{4}$ & 0.16 & 0.09 & 0.08 & 0.32 & 0.37 \\
\hline$A_{5}$ & 0.16 & 0.28 & 0.30 & 0.32 & 0.37 \\
\hline$a_{j}$ & 0.36 & 0.09 & 0.04 & 0.07 & 0.02 \\
\hline$b_{j}$ & 0.03 & & & \\
\hline
\end{tabular}

Table 7. Calculate the Sensivitiy measures: MIRR and LIRR after modification of decision problem given in Table 2

\begin{tabular}{|l|l|l|l|l|l|l|l|l|}
\hline & $C_{1}$ & $C_{2}$ & $C_{2}$ & $C_{4}$ & $C_{5}$ & Rank Order & Change? & Ratio \\
\hline$w_{j}$ & 0.41 & 0.013 & 0.3 & 0.06 & 0.22 & & & \\
\hline$F^{*}(j)=1$ & 1 & 1 & 1 & 1 & 1 & $A_{1} \geq A_{2} \geq A_{4} \geq A_{2} \geq A_{5}$ & & \\
\hline$F^{*}(p)=-1$ & -1 & 1 & 1 & 1 & 1 & $A_{1} \geq A_{5} \geq A_{4} \geq A_{2} \geq A_{2}$ & No & \\
\hline$F^{*}(j)=-1$ & -1 & 1 & -1 & 1 & 1 & $A_{5} \geq A_{2} \geq A_{1} \geq A_{4} \geq A_{2}$ & Yes & $\begin{array}{l}2 \mid 5 \\
\text { (MIRR) }\end{array}$ \\
\hline$F^{*}(p)=-1$ & 1 & 1 & 1 & 1 & 1 & $A_{1} \geq A_{2} \geq A_{4} \geq A_{2} \geq A_{5}$ & No & \\
\hline$F^{*}(p)=-1$ & 1 & -1 & 1 & 1 & 1 & $A_{1} \geq A_{2} \geq A_{4} \geq A_{2} \geq A_{5}$ & No & \\
\hline$F^{*}(p)=-1$ & 1 & -1 & 1 & -1 & 1 & $A_{a} \geq A_{1} \geq A_{4} \geq A_{5} \geq A_{2}$ & Yes & $\begin{array}{l}2 \mid 5 \\
\text { (LIRR) }\end{array}$ \\
\hline
\end{tabular}

MIR Ratio Let $x=|M|$ where $M$ is the MIR criteria set. Then the MIR Ratio is given by $M I R R=x \mid m$ where $m$ is the number of criteria in the decision problem.

LIR Ratio Let $y=|L|$ where $L$ is the LIR criteria set. Then the MIR Ratio is given $L I R R=y \mid m$ where $m$ is the number of criteria in the decision problem.

The MIRR and LIRR are defined in terms of the preferred alternative which results from the S-SAW final ranking. An example of how the MIRR and LIRR are calculated is given in Table 6. The decision data used is given in Table 2.

The MIRR measures how stable the preferred alternative is with respect to minimum values for criteria with the highest weights. The MIRR determines the minimum number of the most important criteria which can be minimized and cause a change in the preferred alternative. The MIRR is given as $x \mid m$ where $\mathrm{x}$ is the smallest number of the most important criteria which caused the change in the preferred alternative. If $\mathrm{x}$ is small relative to $\mathrm{m}$ this means that the preferred alternative depends heavily on criteria with high weights and these criteria need to be measure with a great degree of accuracy. Table 5 shows a MIRR value of $1 \mid 5$ or 0.2 for the decision problem which means that the preferred alternative is very sensitive to $C_{1}$. On the other hand, if MIRR is large this means that the preferred alternative is very stable when there is uncertainty with the most important criteria.

The LIRR measures how stable the preferred alternative is with respect to minimu $m$ values for criteria with the lowest weighs. The LIRR determines the minimum number of the least important criteria which can be minimized and cause a change in the preferred alternative. The LIRR is given as $y \mid m$ where $\mathrm{y}$ is the smallest number of least important criteria which caused the change in the preferred alternative. If LIRR is less than 0.3 this means that the preferred alternative depends heavily on criteria with low weights. This situation means that the preferred alternative cannot survive a large degree of uncertainty and that some other alternative is very close to becoming the optimal alternative. Table 5 shows a LIRR value of 0.8 for the decision problem which means that the preferred alternative is sensitive to the set $\left\{C_{2}, C_{2}, C_{4}, C_{5}\right\}$ since these criteria have the 4 lowest weights. The worse values for $C_{2}, C_{\mathrm{g}}, C_{4}$ and $C_{5}$ will invoke a change in the preferred alternative and therefore the decision maker has to be aware of this so that he will not consider this situation if he wishes to maintain the position of the optimal alternative. This value for the LIRR reinforces the conclusion made from the MIRR value which suggests that the decision problem is very sensitive to $C_{1}$.

Because $C_{1}$ has the the highest weighting does not necess ary makes the results of the above analysis obvious. Consider, Table 6 which shows a slightly modified decision matrix from the one given in Table 2. The new MIRR value is 2|5 or 0.4 for the modified decision problem which means that the preferred alternative $A_{1}$ is not as sensitive to criterion $C_{1}$ (which has the highest weighting) as in the previous decision problem.

This is illustrated in Table 7 and it means that the decision maker can tolerant more uncertainty in $C_{1}$ values. The measurement also suggests that the criteria in the set $\left\{C_{1}, C_{\mathrm{a}}\right\}$ collectively influence the output. 
Similarly, from Table VII the LIRR values for the new decision problem is $2 / 5$ or 0.4 which means that the preferred alternative is sensitive to the set $\left\{C_{2}, C_{\mathrm{a} 4}\right\}$.

\section{S-SAW OBJECTIVE FUNCTION}

The S-SAW objective function is the key for understanding the rationale for using the MIR and LIR measures for SA. It is important at this time to define the concept of a pareto optimal alternative.

A Pareto optimal alternative is an alternative $a$ from a set of alternatives $A$, where there exists no other alternative $x \in A$ such that $f_{j}(a) \leq f_{j}(x)$ for all, $j \in\{1,2, \ldots, m\}$ with at least one strict inequality.

Note that the set of alternatives $A$ may or may not contain a pareto optimal alternative or there may exists a subset $A_{s} \subset A$ of pareto optimal alternatives. Also, $a$ is a weak pareto optimal alternative from a set of alternatives $A$, when there exists no other alternative $x \in A$ such that $f_{j}(a)<f_{j}(x)$ for all, $j \in\{1,2, \ldots, m\}$. In this paper the term pareto optimal is used to represent both situations where $f_{j}(a)<f_{j}(x)$ or $f_{j}(a) \leq f_{j}(x)$ for all, $j \in\{1,2, \ldots, m\}$.

Lemma 1 Given a set of alternatives $A$ the S-SAW method will rank a pareto optimal alternative as the preferred alternative if the objective function $F^{*}(j)=1$ $\forall j \in\{1,2, \ldots m\}$.

Proof. Let $F^{*}(j)=1$ for $\forall j \in\{1,2, \ldots, m\}$ and $y_{h}$ be the maximu $m$ of

$$
y_{i}=\sum_{j=1}^{m} w_{j} z_{i j} F^{*}(j)=\sum_{j=1}^{m} w_{j} z_{i j} \forall i .
$$

Note that $h \in\{1,2, \ldots, n\}$.

If alternative $y_{h}$ is not pareto optimal then there is another alternative $i$ for which $z_{i j} \geq y_{h j} \quad \forall j$ and the inequality is strict for at least one $\mathrm{j}$ then $y_{i}>y_{h}$ which is a contradiction.

As discussed earlier, when $F^{*}(j)=1$ for all criteria $\bar{j}$, the SAW and S-SAW have the same alternative ranking. According to Lemma 1 the preferred alternative would be an optimal alternative. What about when $F^{*}(j)=-1$ for all criteria $j$ ? Would the preferred alternative be ranked last in the rank order of alternatives? Consider Lemma 2.

Lemma 2 Given a set of alternatives $A$ the S-SAW method will rank the preferred alternative as the last alternative in the rank order of alternatives if the objective function $F^{*}(j)=-1 \forall j \in\{1,2, \ldots m\}$.

Proof. Let $F^{*}(j)=-1$ for $\forall j \in\{1,2, \ldots, m\}$ and $y_{h}=\max _{i}\left(\sum_{j=1}^{m} w_{j} z_{i j} F^{*}(j)\right)=-\sum_{j=1}^{m} w_{j} z_{i j}$

From Lemma 1, yh has the highest value and it is the preferred optimal alternative. Therefore, $-y_{h}$ will be the lowest value and so alternative $h \in\{1,2, \ldots, n\}$ will rank the preferred alternative as the last alternative in the rank order of alternatives.

From Lemma 2, the highest possible value of $y_{h}$ intuitively means that all criteria have maximum values for $a_{i j}$ values in the decision table. When $F^{*}(j)=-1$ the explanation of why the least ranked alternative is ranked as the preferred alternative is interesting. When $z_{\mathrm{i} j}<0$ and $F^{*}(j)<0$ then the product of the two will be positive. Hence $w_{j} z_{i j} F^{*}(j) \geq 0$ for all criteria and therefore $0 \leq \sum_{j=1}^{m} w_{j} z_{i j} F^{*}(j) \leq m$.

Lemma 2 justifies why MIR and LIR measures are calculated by first setting $F^{*}(j)=1$ for all criteria. In the case of the MIR the criteria with the highest weights are set to $F^{*}(p)=-1$ where $p \in M$ until the preferred alternative ranking is changed. Each criterion that is minimized will decrease the value of $\sum_{j=1}^{m} w_{j} z_{i j} F^{*}(j)$ relative to the preferred alternative $h \in\{1,2, \ldots, n\}$. Higher $|M|$ (see Section V) means that more important criteria have to be minimized before there is a different preferred alternative. If $|M|$ is small relative to $m$ this means that the preferred alternative depends heavily on the members of $M$ and therefore a great degree of accuracy must be employed for measuring these criteria.

The range of the SAW normalization is $[0,1]$ and that of the S-SAW normalization is $[-1,1]$. The justification for using $[-1,1]$ in the S-SAW is simply to make the range symmetric so that an additive inverse exists for each $w_{j} z_{i j} F^{*}(j) \in \mathbb{R}$ for all $i$ and $i$ values. This means that the operation $\sum_{j=1}^{m} w_{j} z_{i j} F^{*}(j)$ has meaningful addition. However, in the case of the SAW method where normalization has a range of $[0,1]$, the $w_{j} z_{i j}$ product does not have an additive inverse on $[0,1]$. Th is means that the addition operation is compromised.

\section{CONCLUSION}

An extension of the SAW method called Sensitive Simple Additive Weights (S-SAW) method is proposed which allows a decision maker to do sensitivity analysis as a post-optimality step like in linear programming. The method introduces an objective function for criteria and uses this function to minimize or maximize criteria optimization goals. Two quantitative measures: MIRR and LIRR are introduced which allows the decision maker to measure different

sensitivity aspects of the decision problem which informs the decision maker of the degree of risk he should allow for a given criterion or groups of criteria.

By using examples it is shown that the introduced quantitative measures helps the decision maker to determine which criterion or group of criteria influences the stability of the preferred alternative and gives a qualitative insight into which criteria can tolerant uncertainty. Therefore, using the S-SAW approach would help deciaion makers better evualtae risks as sociated with decision criteria.

\section{REFERENCES}

[1] Y.S., Huang, W.C Chang,., W.H., Li, and Z.L. Lin, "Aggregation of utility-based individual preferences for group decision-making", European Journal of Operational Research, vol. 229, no. 2, pp. 462-469. 2013

[2] C. L. Hwang and K. Yoon, "Multiple attribute decision making methods and application", Springer, New York, NY, USA (1981). 
[3] R. Janssen, "Multiobjective decision support for environmental management". Kulwer Academic Publishers, Netherlands (1996)

[4] K. Koffka and W. Goodridge. "Fault Tolerant MultiCriteria Multi-Path Routing in Wireless Sensor Networks." I.J. Intelligent Systems and Applications, 06, pp. 55-63, 2015

[5] K. Koffka and W. Goodridge. "Energy Aware Ad Hoc On-Demand Multipath Distance Vector Routing." I.J. Intelligent Systems and Applications, 07, pp. 50-56, 2015

[6] A. Afshari, M. Mojahed and R. M. Yusu, "Simple additive weighting approach to personnel selection problem, International Journal of Innovation", Management and Technology, vol 1(5), pp. 511-515, 2010

[7] L. Abdullah and A. Otheman. "A New Entropy Weight for Sub-Criteria in Interval Type-2 Fuzzy TOPSIS and Its Application." I.J. Intelligent Systems and Applications, vol. 02 , pp.25-33, 2013,

[8] M. Behzadian, S. Otaghsara, M. Yazdani and J. Ignatius. "A state-of-the-art survey of TOPSIS applications". Expert Systems with Applications, 39(17): pp.1305113069, 2012.

[9] D. Stanujkic, B. Djordjevic, and M. Djordjevic, "Comparative analysis of some prominent MCDM methods: A case of ranking Serbian banks", Serbian Journal of Management, vol. 8, No. 2, pp. 213-241, 2013.

[10] W. Deni, O. Sudana and A. Sasmitaf, "Analysis and implementation fuzzy multiattribute decision making saw method for selection of high achieving students in faculty level", International Journal of Computer Science, vol. 10(1), pp. 674-680, 2013.

[11] J. Barzilai and F. Lootsma, "Power relations and group aggregation in the multiplicative ahp and smart", Journal of Multi-Criteria Decision Analysis vol. 6(3) , pp. 155165,1997

[12] J.-J. Wang, Y.-Y. Jing, C.-F. Zhang and J.-H. Zhao, Review on multi-criteria decision Analysis aid in sustainable energy decision-making, Renewable and Sustainable Energy,Reviews 13(9) (2009) 2263-2278.

[13] S. Sitarz, "Multi-Criteria Analysis, Sensitivity Analysis, Multi-Objective Linear Programming, Decision Making", I.J. Intelligent Systems and Applications, vol. 5, No. 3, pp. 50-57, 2013.

[14] E. Triantaphyllou and A. Sanchez, A sensitivity analysis approach for some deterministic Multi-criteria decisionmaking methods*, Decision Sciences 28(1) (1997) 151194.

[15] B. Oztaysi, T. Kaya and C. Kahraman, "Performance comparison based on customer relationship management using analytic network process", Expert Systems with Applications, vol. 38(8), pp. 9788-9798, 2011

[16] W. Wolters and B. Mareschal, Novel types of sensitivity analysis for additive mcdm methods, European Journal of Operational Research 81(2) (1995) 281-290

[17] B. Feizizadeh, P. Jankowski and T. Blaschke, "A gis based spatially-explicit sensitivity and uncertainty analysis approach for multi-criteria decision analysis", Computers \& Geosciences, vol. 64, pp. 81-95, 2014

[18] R. Simanaviciene and L. Ustinovichius, Sensitivity analysis for multiple criteria decision making methods: TOPSIS and SAW, Procedia - Social and Behavioral Sciences 2(6) (2010) 7743-7744, Sixth International Conference on Sensitivity Analy sis of Model Output.

[19] J. Butler, J Jia and J. Dyer, "Simulation techniques for the sensitivity analysis of multicriteria decision models", European Journal of Operational Research, 103 (3), pp. 531-546. doi: 10.1016/S0377-2217(96)00307-4, 1997
[20] L Yu, K K, Lai “A distance-based group decision-making methodology for multi-person multi-criteria emergency decision support", Decision Support Systems, Vol.51, No.2,pp. 307-315,2011"

[21] C.A. Bana e Costa. A methodology for sensitivity analy sis in three-criteria problems: a case study in municipal management. European Journal of Operational Research, 33:159-173, 1988.

[22] T. L. Saaty, "A scaling method for priorities in hierarchical structures", Journal of Mathematical Psychology vol. 15, pp. 57-68, 1977.

\section{Authors' Profiles}

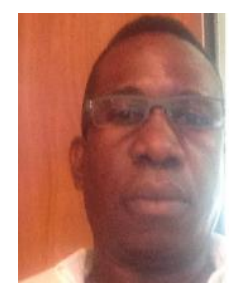

Wayne Goodridge is a Lecturer in the Department of Computing and Information Technology, The University of the West Indies, St. Augustine. He did his $\mathrm{PhD}$ at Dalhousie University and his research interest includes computer communications and security

How to cite this paper: Wayne S. Goodridge, "Sensitivity Analysis Using Simple Additive Weighting Method", International Journal of Intelligent Systems and Applications (IJISA), Vol.8, No.5, pp.27-33, 2016. DOI: 10.5815/ijisa.2016.05.04 